\title{
Precise Analytical Solution for the Peak Gain of LLC Resonant Converters
}

\author{
Sung-Soo Hong*, Sang-Ho Cho*, Chung-Wook Roh*, and Sang-Kyoo Han ${ }^{\dagger}$ \\ ${ }^{\dagger *}$ Dept. of Electrical Engineering, Kookmin University, Seoul, Korea
}

\begin{abstract}
Although LLC resonant converters have the advantages of a wide operation range and high efficiency, the lack of an analytical solution for the peak gain makes it difficult to optimize the resonant tank design, when considering not only the normal condition but also the holdup time requirement. In this paper, based on a mathematical analysis of a LLC resonant converter at the peak gain point, an analytical solution for the peak gain has been developed. By using the developed analytical solution, the peak gain with given resonant tank parameters can be obtained. To confirm the validity of the developed analytical solution, simulations and experimental results are compared.
\end{abstract}

Key Words: Holdup time, LLC resonant converter, Mathematical analysis, Peak gain

\section{INTRODUCTION}

With the development of power conversion technology, power density has become a major challenge for the switching power supply [1]-[3]. To improve power density, a higher switching frequency and efficiency are required. A higher switching frequency results in a volume reduction in the passive components. High efficiency can reduce the volume for thermal management [4],[5].

In addition to a high switching frequency and efficiency, a switching power supply requires a holdup time specification in many electronic systems. To satisfy the holdup time requirement, a switching power supply should keep up its output voltage even at the minimum input voltage condition. In other words it needs to be designed with a wide input range. Apparently, a switching power supply operating in a wide input range can reduce the DC link capacitance size and improve the power density.

Among the many topologies, a LLC resonant converter becomes the most attractive topology for medium power applications due to its high efficiency and wide input range. When the switching frequency equals the resonant frequency, the LLC resonant converter operates optimally in terms of efficiency. Under this condition, the conduction loss and the switching loss can be minimized. According to the circuit operation analysis in [6], because the impedance of a resonant tank becomes zero at the resonant frequency, the input and output voltages are virtually connected together. Hence, the LLC resonant converter can be easily designed with the unity gain at the resonant frequency.

\footnotetext{
Manuscript received Jul. 16, 2010; revised Sep. 28, 2010

$\dagger$ Corresponding Author: djhan@kookmin.ac.kr

Tel: +82-2-910-5467, Fax: +82-2-910-4449, Kookmin Univ.

* Dept. of Electrical Engineering, Kookmin University, Korea
}

On the other hand, from a holdup time point of view, it is important to design a resonant tank, considering not only normal conditions but also minimum input voltage conditions.

However, until now, research on LLC resonant converters has mostly focused on the normal condition designs in [5][8] and there is no precise mathematical analysis of the peak gain on the holdup time requirement due to its complexity. Apparently, the only way to design a resonant tank considering the holdup time requirement is the trial and error method, which takes more time and design resources.

Meanwhile, to simplify the analysis, the peak gains at different $L_{n}$ and $Q$ combinations are simulated based on the simulation tool "Simplis" in [9], which can get peak gain curves for different $L_{n}$ and $Q$ values with a short simulation time. In a set of curves, each line shows the combination of different $L_{n}$ and $\mathrm{Q}$ values that can achieve the same peak gain. Thus, the computer-based method provides a simple design guideline and can reduce the number of trial and error attempts. However, this method does not take sets of series $L_{n}$ and $Q$ combinations into consideration. Therefore, it is less accurate than an analytical solution.

In this paper, to overcome the above mentioned limitations when the LLC resonant converter is operating at the peak gain point, a mathematical analysis of the peak gain is presented with given set of parameters such as the resonant capacitor, the resonant inductor, the magnetizing inductor, and the turn ratio of the transformer obtained by the methods presented in the literature mentioned above. By using the presented mathematical analysis method for the peak gain, it is possible to optimize the resonant tank of a LLC resonant converter, which can satisfy the holdup time requirement with less design resource in a relatively short time.

To confirm the validity of the developed analytical solution, 


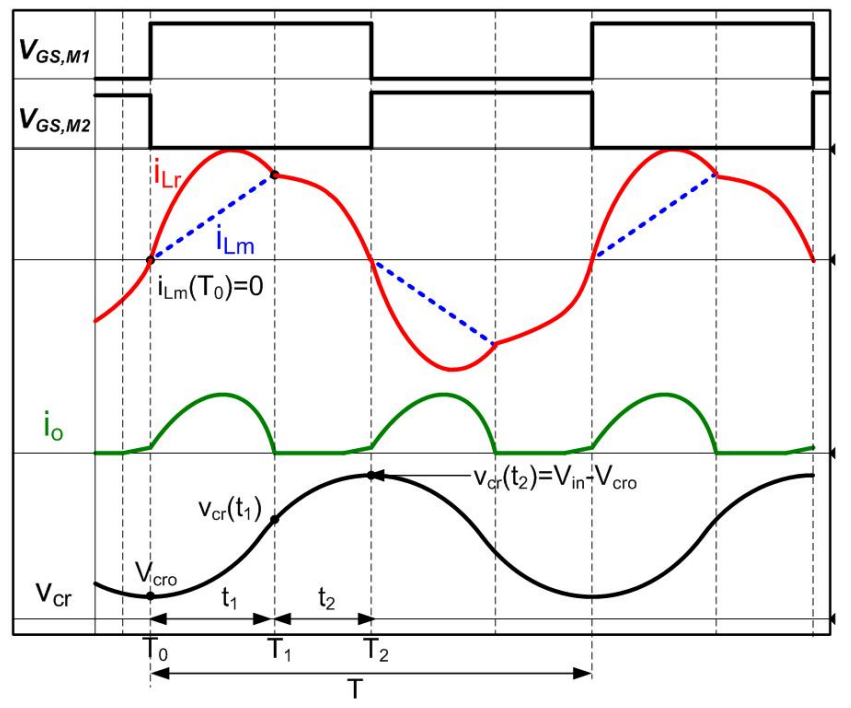

Fig. 1. Key waveforms of LLC resonant converter at peak gain point.

experimental results and simulations are compared.

\section{Theoretical Analysis of the PeAK Gain for LLC RESONANT CONVERTERS}

The peak gain happens when a circuit is operating at the boundary of zero current switching (ZCS) and zero voltage switching (ZVS) modes. A resonant tank current under this condition is shown in Figure 1. In each half switching cycle, the magnetizing inductor is increased by the output voltage. After the magnetizing inductor current equals the resonant inductor current, the magnetizing inductor participates in the resonance and the magnetizing energy is transferred to the resonant capacitor. When the resonant current is reset to zero at the end of each half of a switching cycle, the entire energy stored in both the resonant inductor and the magnetizing inductor will be transferred to the resonant capacitor, and hence the voltage conversion ratio reaches its peak value.

In this paper, based on the operation principle mentioned above, a mathematical analysis of the peak gain has been presented.

\section{A. Mathematical analysis for LLC resonant converters}

Mode $1\left[T_{0} \sim T_{1}\right]$ : When $M_{2}$ is turned off and $M_{1}$ is turned on at $T_{o}$, Mode 1 begins as shown in Figure 1. Initially, the resonant tank current is zero. During this period, the difference between the resonant tank current and the magnetizing current is transferred to the secondary side as shown in Figure 2(a). When the resonant inductor current $i_{L r}$ becomes equal to the magnetizing current $i_{L m}$ at $T_{1}$, Mode 1 ends. The equivalent circuit in Mode 1 can then be shown as in Figure 2(b). The voltage $v_{c r}$ across $C_{r}$, the current $i_{L r}$ through $L_{r}$, and the current $i_{L m}$ through $L_{m}$ at the end of this mode can be expressed at $T_{1}$ as a function of the time duration $t_{1}$ of Mode 1 as:

$$
\begin{gathered}
v_{c r}\left(t_{1}\right)=\left(V_{i n}-n V_{o}\right)\left\{1-\cos \left(\omega_{o} t_{1}\right)\right\}+V_{c r o} \cos \left(\omega_{o} t_{1}\right) \\
i_{L r}\left(t_{1}\right)=\sqrt{\frac{C_{r}}{L_{r}}}\left(V_{\text {in }}-n V_{o}-V_{\text {cro }}\right) \sin \left(\omega_{o} t_{1}\right)
\end{gathered}
$$

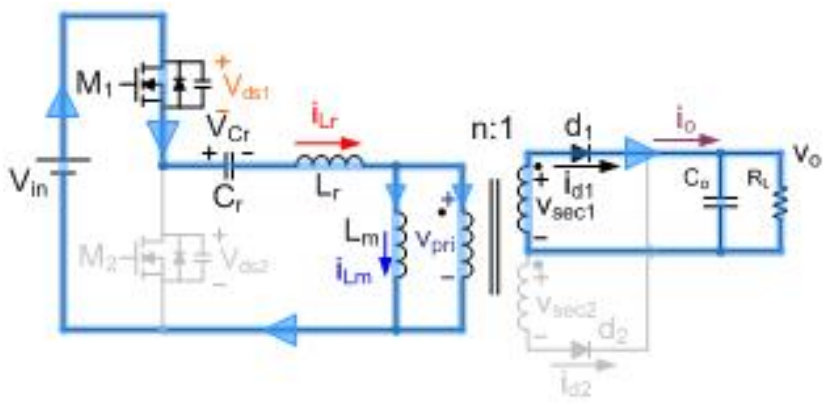

(a) Topological stage of mode analysis at Mode 1 .

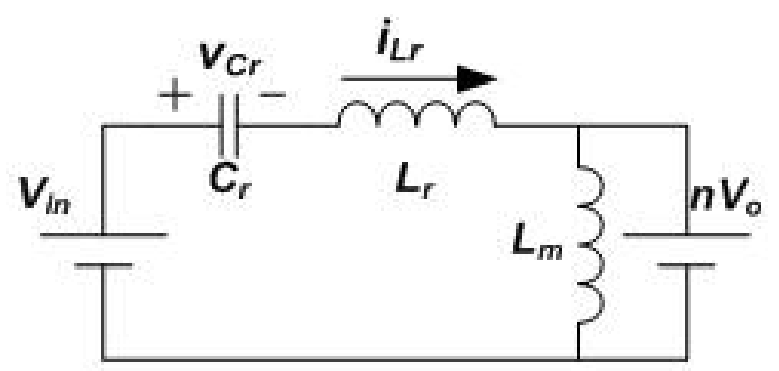

(b) The equivalent circuit for working at Mode 1.

Fig. 2. Operation mode analysis at Mode 1.

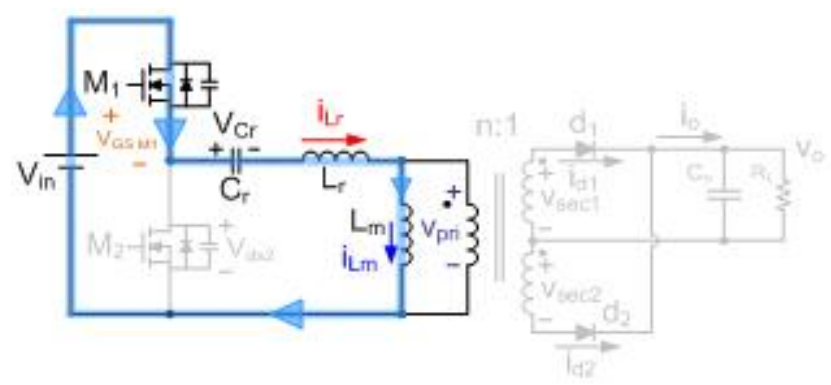

(a) Topological stage of mode analysis at Mode 2.

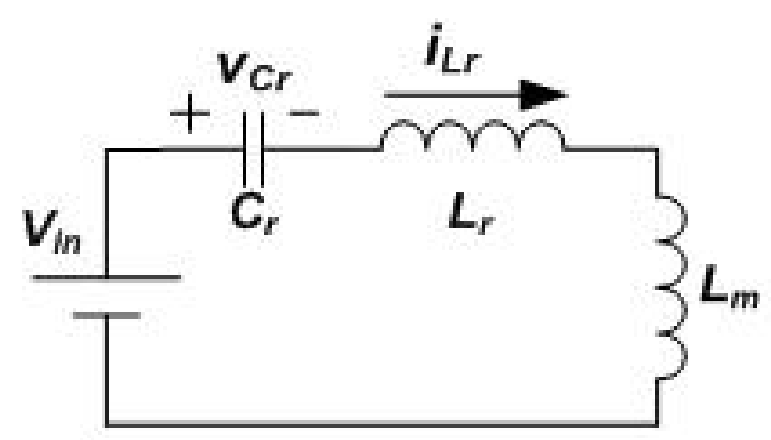

(b) The equivalent circuit for working at Mode 2 .

Fig. 3. Operation mode analysis at Mode 2.

$$
i_{L m}\left(t_{1}\right)=\frac{n V_{o}}{L_{m}} t_{1}
$$

where $V_{c r o}$ is the initial value of $v_{c r}$ at $T_{0}$, as shown in Figure 1 and the resonant frequency $\omega_{o}$ becomes:

$$
\omega_{0}=\frac{1}{\sqrt{L_{r} C_{r}}}
$$

Mode 2[ $\left.T_{1} \sim T_{2}\right]$ : For the convenience of analysis, the time 
from $T_{1}$ to $T_{2}$ is newly defined as $t_{2}$. When the current $i_{L r}$ becomes equal to the current $i_{L m}$ and the secondary side current reaches zero, the secondary rectifier diode is blocked and then Mode 2 begins as shown in Figure 3(a). The equivalent circuit for Mode 2 can then be shown as in Figure 3(b). The voltage $v_{c r}$ and current $i_{L r}$ during this period can be expressed as a function of the time duration $t_{2}$ of this mode as:

$$
\begin{gathered}
v_{C r}\left(t_{2}\right)=V_{i n}\left(1-\cos \left(\omega_{1} t_{2}\right)\right)+v_{C r}\left(t_{1}\right) \cos \left(\omega_{1} t_{2}\right) \\
+i_{L r}\left(t_{1}\right) \sqrt{\frac{L_{e q}}{C_{r}}} \sin \left(\omega_{1} t_{2}\right) \\
i_{L r}\left(t_{2}\right)=\sqrt{\frac{C_{r}}{L_{e q}}}\left(V_{i n}-V_{c r}\left(t_{1}\right)\right) \sin \left(\omega_{1} t_{2}\right)+i_{L r}\left(t_{1}\right) \cos \left(\omega_{1} t_{2}\right)
\end{gathered}
$$

where $L_{e q}$ is the sum of $L_{r}$ and $L_{m}$, and the resonant frequency including the magnetizing inductance $\omega_{1}$ becomes:

$$
\omega_{1}=\frac{1}{\sqrt{\left(L_{r}+L_{m}\right) C_{r}}}
$$

Meanwhile, the current $i_{L r}$ through $L_{r}$ is equal to zero at $t_{2}$. Therefore, equation (6) can be expressed as follows:

$$
\sqrt{\frac{C_{r}}{L_{e q}}}\left(V_{i n}-v_{c r}\left(t_{1}\right)\right) \sin \left(\omega_{1} t_{2}\right)+i_{L r}\left(t_{1}\right) \cos \left(\omega_{1} t_{2}\right)=0
$$

Since the resonant capacitor acts as a DC blocking capacitor, the DC offset of the resonating capacitor voltage is half of the input voltage. Then, the resonant capacitor voltage at the end of Mode 2 can be obtained as:

$$
v_{c r}\left(t_{2}\right)=V_{\text {in }}-V_{\text {cro }}
$$

From equations (5) and (9), the initial voltage $V_{\text {cro }}$ can be obtained as follows:

$V_{\text {cro }}=V_{\text {in }} \cos \left(\omega_{1} t_{2}\right)-v_{c r}\left(t_{1}\right) \cos \left(\omega_{1} t_{2}\right)-i_{L r}\left(t_{1}\right) \sqrt{\frac{L_{e q}}{C_{r}}} \sin \left(\omega_{1} t_{2}\right)$.

\section{B. Peak gain of LLC resonant converters}

Until now, five equations, as shown in equations (1), (2), (3), (8), and (10), have been derived from the equivalent circuit with the given parameters, $V_{i n}, R_{L}, L_{m}, L_{r}, C_{r}$, and $n$, when the LLC converter is operating at the peak gain point. Since the resonant inductor current $i_{L r}\left(t_{1}\right)$ becomes equal to the magnetizing current $i_{L m}\left(t_{1}\right)$ at $t_{1}$, these equations have the following six unknown values: $t_{1}, t_{2}, i_{L r}\left(t_{1}\right), v_{c r}\left(t_{1}\right), V_{c r o}$, and $v_{o}$. Thus, to solve for the six unknown values, an additional equation is required.

The difference between the resonant inductor current and the magnetizing current during the Mode 1 interval is transferred to the load. Therefore, the following equation holds:

$$
\frac{2}{T} \int_{0}^{t 1}\left\{i_{L r}(t)-i_{L m}(t)\right\} d t=\frac{V_{o}}{n R_{L}}
$$

From equations (2), (3), and (11), the output voltage $V_{o}$ leads to:

$$
V_{o}=\frac{C_{r}\left(V_{\text {cro }}-V_{\text {in }}\right)\left\{\cos \left(\omega_{o} t_{1}\right)-1\right\}}{\frac{\left(t_{1}+t_{2}\right)}{2 n R_{L}}-n\left\{C_{r}\left(\cos \left(\omega_{o} t_{1}\right)-1\right)-\frac{n t_{1}^{2}}{2 L_{m}}\right\}} .
$$

To eliminate $i_{L r}\left(t_{1}\right)$ and $v_{c r}\left(t_{1}\right)$, substituting equations (1) and (2) into equation (8) results in:

$=\frac{\left(V_{\text {in }}-V_{\text {cro }}\right)\left\{\sqrt{\frac{C_{r}}{L_{e q}}} \cos \left(\omega_{o} t_{1}\right) \sin \left(\omega_{1} t_{2}\right)+\sqrt{\frac{C_{r}}{L_{r}}} \sin \left(\omega_{o} t_{1}\right) \cos \left(\omega_{1} t_{2}\right)\right\}}{n\left\{\sqrt{\frac{C_{r}}{L_{r}}} \sin \left(\omega_{o} t_{1}\right) \cos \left(\omega_{1} t_{2}\right)-\sqrt{\frac{C_{r}}{L_{e q}}}\left(1-\cos \left(\omega_{o} t_{1}\right)\right) \sin \left(\omega_{1} t_{2}\right)\right\}}$.

In the same manner, substituting equations (1) and (2) into equation (10) yields:

$\frac{\left.V_{\text {in }}-V_{\text {cro }}\right)\left\{\cos \left(\omega_{o} t_{1}\right) \cos \left(\omega_{1} t_{2}\right)+\sqrt{\frac{L_{e q}}{L_{r}}} \sin \left(\omega_{o} t_{1}\right) \cos \left(\omega_{1} t_{2}\right)\right\}-V_{c r o}}{n\left\{\cos \left(\omega_{o} t_{1}\right) \cos \left(\omega_{1} t_{2}\right)-\cos \left(\omega_{1} t_{2}\right)-\sqrt{\frac{L_{e q}}{L_{r}}} \sin \left(\omega_{o} t_{1}\right) \sin \left(\omega_{1} t_{2}\right)\right\}}$

Meanwhile, the current $i_{L r}\left(t_{1}\right)$ is equal to the current $i_{L m}\left(t_{1}\right)$ at $t_{1}$. Therefore, from equations (2) and (3), the output voltage $v_{o}$ can be obtained as follows:

$$
V_{o}=\frac{V_{\text {in }}-V_{\text {cro }}}{n\left\{\frac{1}{L_{m}} \sqrt{\frac{L_{r}}{C_{r}}} \frac{t_{1}}{\sin \left(\omega_{o} t_{1}\right)}+1\right\}} .
$$

From equation (15), the peak gain $G_{\text {peak }}$ leads to:

$$
G_{\text {peak }}=\frac{V_{o}}{V_{\text {in }}}=\frac{1-V_{\text {cro }} / V_{\text {in }}}{n\left\{\frac{1}{L_{m}} \sqrt{\frac{L_{r}}{C_{r}}} \frac{t_{1}}{\sin \left(\omega_{o} t_{1}\right)}+1\right\}} .
$$

Equation (15) shows that the output voltage $V_{o}$ can be calculated by determining $V_{\text {cro }}$ and $t_{1}$. The voltage $V_{\text {cro }}$ can be obtained from equations (14) and (15) as follows:

$$
\begin{aligned}
& V_{\text {cro }}=\left(\cos \left(\omega_{o} t_{1}\right) \cos \left(\omega_{1} t_{2}\right)-\sqrt{\frac{L_{e q}}{L_{r}}} \sin \left(\omega_{o} t_{1}\right) \cos \left(\omega_{1} t_{2}\right)\right) \\
& \times\left(1-\left(\frac{1}{L_{m}} \sqrt{\frac{L_{r}}{C_{r}}} \frac{t_{1}}{\sin \left(\omega_{o} t_{1}\right)}\right)^{-1}\right)+\frac{L_{m} \sin \left(\omega_{o} t_{1}\right) \cos \left(\omega_{1} t_{2}\right)}{\sqrt{\frac{L_{r}}{C_{r}}}} t_{1}+L_{m} \sin \left(\omega_{o} t_{1}\right)
\end{aligned}
$$

From equation (17), $V_{\text {cro }}$ can be calculated by determining $t_{1}$ and $t_{2}$.

Meanwhile, by eliminating $V_{o}$ and $V_{\text {cro }}$ from the equations (12), (13), and (15), $t_{1}$ and $t_{2}$ can be solved. In order to get $t_{1}$, it is required that $t_{2}$ is expressed as a function of $t_{1}$. From equations (12) and (15), equation (18) can be derived as:

$$
t_{2}=n^{2} R_{L}\left\{C_{r}\left(1-\cos \left(\omega_{o} t_{1}\right)\left(\frac{1}{L_{m}} \sqrt{\frac{L_{r}}{C_{r}}} \frac{t_{1}}{\sin \left(\omega_{o} t_{1}\right)}\right)-\frac{t_{1}^{2}}{2 L_{m}}\right\}-t_{1} .\right.
$$

Similarly, from equations (13) and (15), equation (19) can be derived as:

$t_{2}=\frac{1}{\sqrt{L_{e q} C_{r}}}\left[\tan ^{-1}\left\{\frac{-\frac{1}{L_{m} \sqrt{C_{r}}} t_{1}}{\frac{1}{\sqrt{L_{e q}}}\left(\frac{1}{L_{m}} \sqrt{\frac{L_{r}}{C_{r}}} \frac{t_{1}}{\sin \left(\omega_{o} t_{1}\right)} \cos \left(\omega_{o} t_{1}\right)+1\right)}\right\}\right]$.

Eliminating $t_{2}$ from equations (18) and (19) gives:

$$
\begin{aligned}
& n^{2} R_{L}\left\{C_{r}\left(1-\cos \left(\omega_{o} t_{1}\right)\left(\frac{1}{L_{m}} \sqrt{\frac{L_{r}}{C_{r}}} \frac{t_{1}}{\sin \left(\omega_{o} t_{1}\right)}\right)-\frac{t_{1}^{2}}{2 L_{m}}\right\}-t_{1}\right. \\
& =\frac{1}{\sqrt{L_{e q} C_{r}}}\left[\tan ^{-1}\left\{\frac{-\frac{1}{L_{m} \sqrt{C_{r}}} t_{1}}{\frac{1}{\sqrt{L_{e q}}}\left(\frac{1}{L_{m}} \sqrt{\frac{L_{r}}{C_{r}}} \frac{t_{1}}{\sin \left(\omega_{o} t_{1}\right)} \cos \left(\omega_{o} t_{1}\right)+1\right)}\right\}\right] .
\end{aligned}
$$


As shown in equation (20), this equation contains only one unknown value $t_{1}$ while all of the other parameters are given values. Unfortunately, it is difficult to derive a solution of $t_{1}$ as a closed form from equation (20). However, the time $t_{1}$ can be easily calculated numerically and iteratively by using a computer-based tool. Then, time $t_{2}$ can be calculated by either equation (18) or (19).

Once $t_{1}$ and $t_{2}$ are found, then the switching frequency at the peak gain point can be expressed as follows:

$$
f_{\text {peakgain }}=\frac{1}{2\left(t_{1}+t_{2}\right)} \text {. }
$$

To sum up, there are four steps to getting the peak gain of the LLC resonant converter. With known parameters, the first step is to calculate the time $t_{1}$ from equation (20). The next step is to calculate the time $t_{2}$ by substitution of the $t_{1}$ value into either equation (18) or (19). The third step is to calculate $V_{\text {cro }}$ by substitution of the $t_{1}$ and $t_{2}$ values into equation (17). The last step is to obtain the peak gain by substitution of the $t_{1}$ and $V_{\text {cro }}$ values into equation (16).

\section{Optimal design considerations}

The peak gain equations (16) and (17) are so complex and messy that it is hard to predict the peak gain characteristic under the parameter variation conditions. Therefore, in this section, graphical simulations are presented to easily design the resonant tank parameters, which can get a large voltage gain. Moreover, some optimal design considerations are explained in this section.

i) As shown in equation (16), in order to get a large voltage gain, a small initial value of the resonant capacitor voltage, $V_{\text {cro }}$ is required. Therefore, a larger resonant capacitance $C_{r}$ can get a higher voltage gain as shown in Figure 4(a).

With a larger $C_{r}$, the voltage stress on $C_{r}$ will be smaller. However, the problem is that the impedance of the resonant tank will be small too, which will affect the short circuit performance. With a smaller tank impedance, there will be a higher short circuit current, and a higher switching frequency is needed to limit the short circuit current [10].

ii) At the end of each half switching cycle, the entire energy stored in both the resonant inductor and the magnetizing inductor, $\left(L_{m}+L_{r}\right) i_{L m}^{2} / 2$, will be transferred to the resonant capacitance $C_{r}$. Therefore, a large voltage gain can be achieved under the small magnetizing inductance $L_{m}$ condition due to the large magnetizing inductor current as shown in Figure 4(b). Similarly, a large voltage gain can be obtained under a large resonant inductance $L_{r}$ under an identical magnetizing inductor current condition as shown in Figure 4(c). Even though a narrow switching frequency range can be achieved due to the small magnetizing inductance, the switching and conduction losses are increased due to the high magnetizing current, which results in poor efficiency.

A large resonant inductance will decrease the switching frequency range of a LLC resonant converter. However, a large resonant inductance makes it difficult to use the transformer leakage inductance, which results in low power density and heat generation.

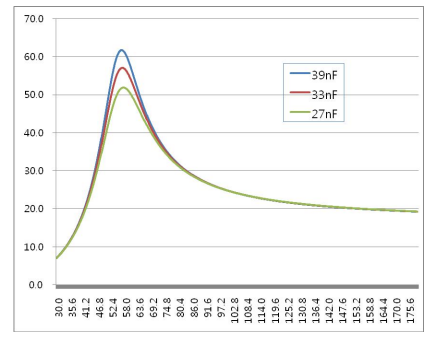

(a) $C_{r}$ variation.

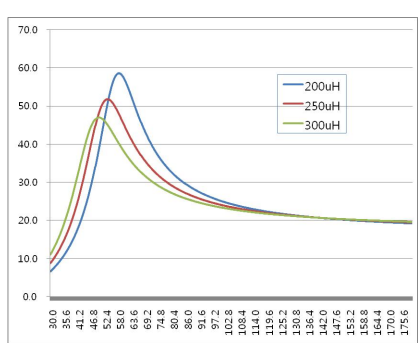

(b) $L_{m}$ variation.

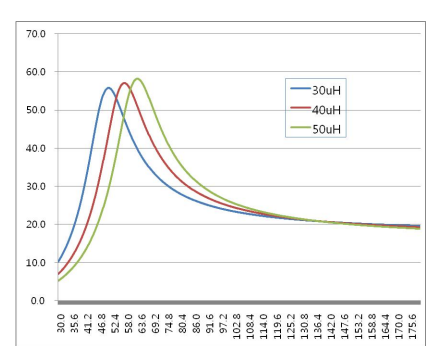

(c) $L_{r}$ variation
Fig. 4. DC gain characteristic under the parameter variation conditions.

TABLE I

DESIGN PARAMETERS FOR SIMULATION

\begin{tabular}{l|c}
\hline \hline Input voltage, $V_{i n}$ & $250[\mathrm{~V}]$ \\
\hline Output voltage, $v_{o}$ & $55[\mathrm{~V}]$ \\
\hline Load resistance, $R_{L}$ & $6.7[\Omega]$ \\
\hline Turn ratio, $n$ & 3.6 \\
\hline Magnetizing inductance, $L_{m}$ & $210[\mathrm{uH}]$ \\
\hline Resonant inductance, $L_{r}$ & $40[\mathrm{uH}]$ \\
\hline Resonant capacitance, $C_{r}$ & $33[\mathrm{nF}]$ \\
\hline \hline
\end{tabular}

\section{Measurement Results}

To confirm the validity of the developed analytical solution, a $450 \mathrm{~W}$ prototype was built with parameters in Table I.

With the given parameters, we can obtain $t_{1}=3.218[\mathrm{us}]$, and $t_{2}=3.472[u s]$ by solving the equation using a computer-based program, which results in $f_{s w}=74.738 \mathrm{kHz}$ at the peak gain point.

A simulation was done with PSIM as shown in Figure 5. Figure 6 shows the key waveforms such as the resonant tank current and the output voltage. As shown in Figure 6(a), the LLC resonant converter gain can be kept constant at the resonant frequency, which results in a LLC resonant converter that can achieve maximum efficiency under normal operation conditions.

During the hold up time, to maintain a regulated output voltage, the switching frequency of the LLC resonant converter needs to be reduced so that the converter gain can be boosted up. As shown in Figure 6(b), the switching frequency of the LLC resonant converter is reduced from about $136 \mathrm{kHz}$ to $74 \mathrm{kHz}$, the resonant tank current becomes zero at the end of Mode 2, and the intervals of Mode 1 and 2 agree well with the mathematical results presented in this paper. Also, the output voltage is accurately regulated at $55[\mathrm{~V}]$ as expected.

Figure 7 shows the experimental set up of a LLC resonant converter. The experimental waveforms under normal operation and at the peak gain point are shown in Figure 8. As shown in Figure 8(a), during normal operation conditions, the LLC resonant converter is operating at its resonant frequency 


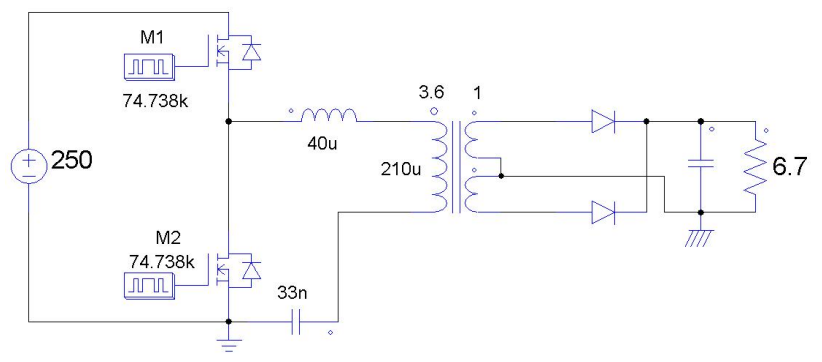

Fig. 5. Simulation circuit.
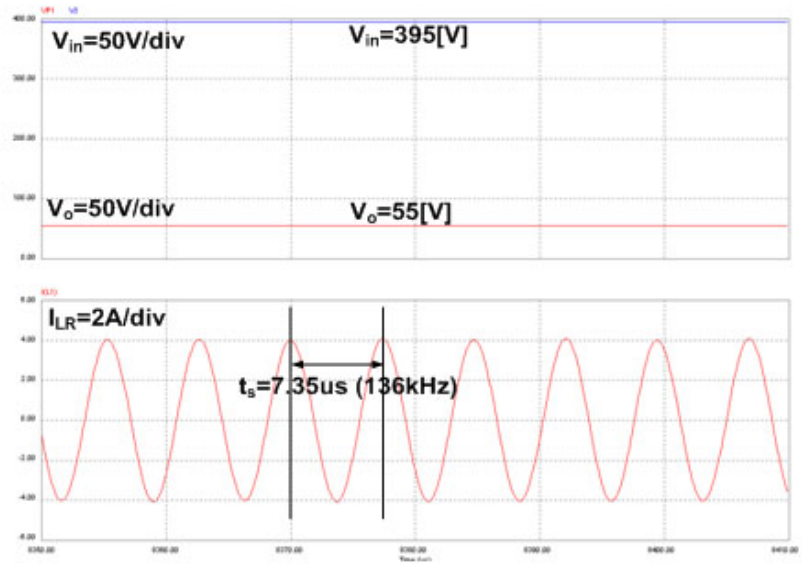

(a) Normal operation.

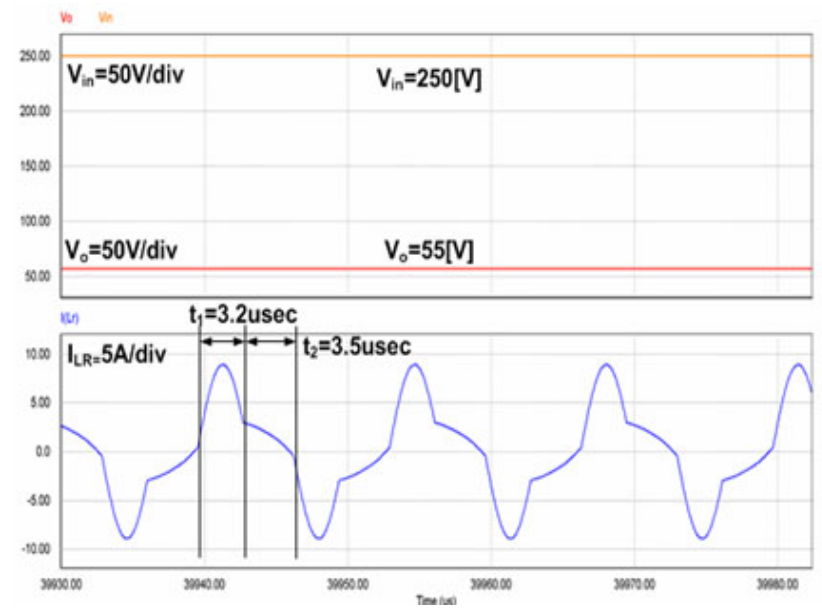

(b) Hold-up time operation.

Fig. 6. Simulation results for proposed mathematical analysis.

to regulate the output voltage. As shown in Figure 8(b), the switching frequency at the peak gain point becomes $74.63[\mathrm{kHz}]$ and the input voltage is equal to $260[\mathrm{~V}]$ for an output voltage of 55[V]. The input voltage and the switching frequency of the simulation results are a little different from those of the experimental results while yielding the same output voltage. This is because circuit parasitics are not considered in the simulation. However, overall the experimental and simulation data have a good agreement.

\section{CONCLUSions}

Smaller switching losses and wide input range operation makes LLC resonant converters attractive as front-end AC/DC

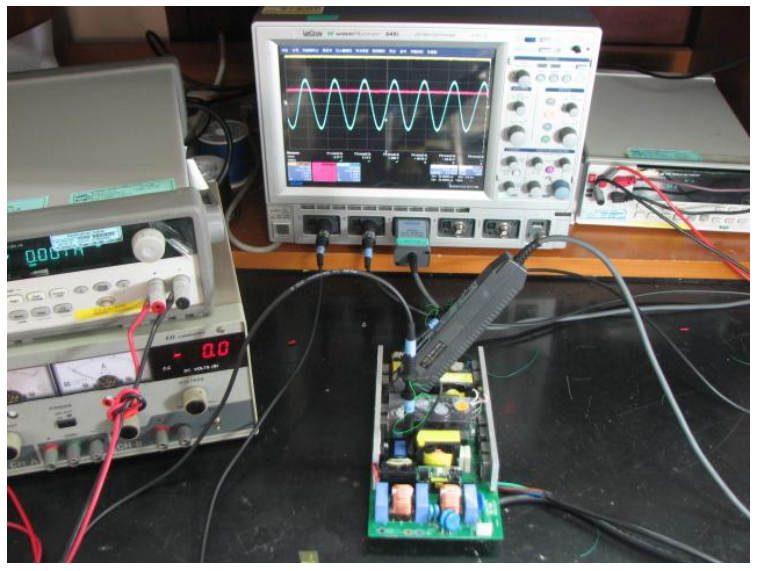

Fig. 7. The experimental set up of the LLC resonant converter.

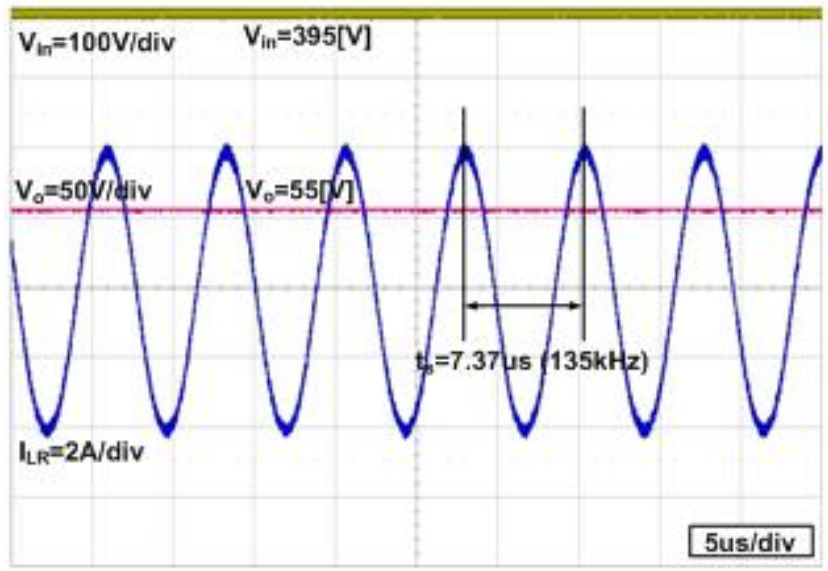

(a) Normal operation.

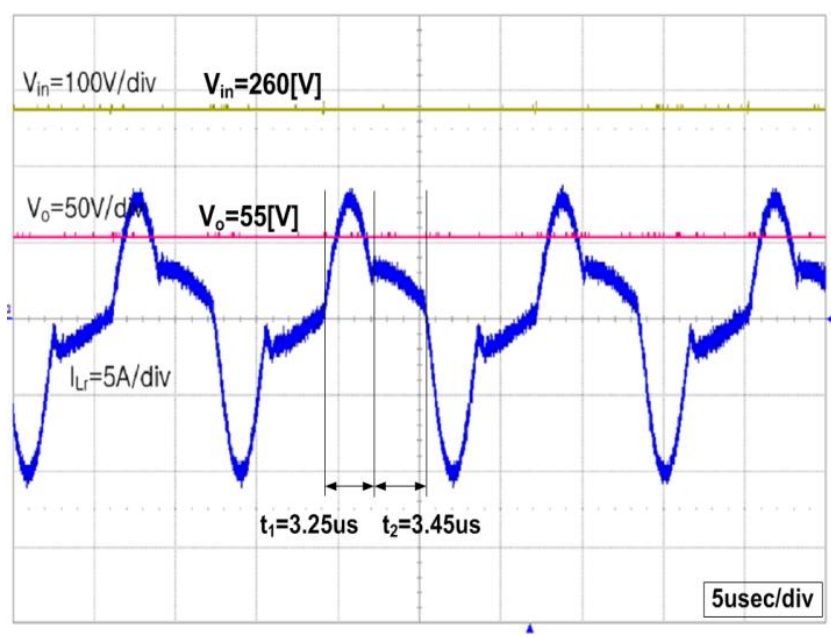

(b) Hold-up time operation.

Fig. 8. Experimental results for proposed mathematical analysis.

converters. However, the lack of an analytical solution for the peak gain makes the design of an optimal resonant tank which can satisfy the holdup time requirement difficult for engineers.

In this paper, based on a theoretical analysis of circuit operation at the peak gain point, a precise analytical solution for the peak gain has been presented. By using the developed precise analytical solution for the peak gain, it is possible 
to accurately optimize the resonant tank of a LLC resonant converter, which can regulate output voltage during hold up time.

The simulations and experimental results of the derived equations for the peak gain of a LLC resonant converter show a good agreement. This means that a LLC resonant converter can be more easily designed with the mathematical equations presented in this paper.

\section{ACKNOWLEDGMENT}

This work was supported (in part) by the New Faculty Research Program 2007 of Kookmin University, Korea and the MKE (The Ministry of Knowledge Economy), Korea, under the ITRC (Information Technology Research Center) support program supervised by the NIPA (National IT Industry Promotion Agency) (NIPA-2010-C1090-1021-0005)

\section{REFERENCES}

[1] B. R. Mower, "SSI: building compliant power elements for servers," in IEEE-APEC Proc., pp.23-27, 1999.

[2] J. D. van Wyk, F. C. Lee, D. Boroyevich, Z. Liang, and K. Yao, "A future approach to integration in power electronics system," IEEE-IECON '03, Vol. 1, pp. 1008-1019, 2003

[3] F. C. Lee, J. D. van Wyk, D. Boroyevich, G. Lu, Z. Liang, and P. Barbosa, "Technology trends toward a system-in- a- module in power electronics," IEEE Circuits and Systems Magazine, Vol. 2, Issue 4, pp. 4-22, 2002.

[4] Y. Gu, Z. Lu, and Z. Qian, "DC/DC topology selection criterion," in Proc. IPEMC, pp. 508-512, 2004.

[5] B. Yang, "Topology investigation for front end DC/DC power conversion for distributed power system," PhD thesis, Virginia Polytechnic Institute and Stage University, 2003.

[6] B. Yang, F. C. Lee, A. J. Zhang, and G. Haung, "LLC resonant converter for front end DC/DC conversion," in Proc. IEEE APEC, pp. 1108-1112, 2002.

[7] T. Liu, Z. Zhou, A. Xiong, J. Zeng, and J. Ying, "A novel precise design method for LLC resonant converter," INTELEC '06, pp. 1-6, 2006.

[8] J-H. Jung and J-G. Kwon, "Theoretical analysis and optimal design of LLC resonant converter," Power electronics and applications, pp. 1-10, Jan. 2007.

[9] B. Lu, W. Liu, Y. Liang, F. C. Lee, and J. D. van Wyk, "Optimal design methodology for LLC resonant converter," in Proc. IEEE APEC, pp.533538, Apr. 2006.

[10] B. Yang, "Topology Investigation for Front End DC/DC Power Conversion for Distributed Power System," Doctor Philosophy in Electrical Engineering, Virginia Polytechnic Institute and State University, Sept. 2003.

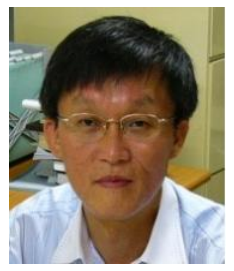

Sung-Soo Hong received his B.S. in Electrical Engineering from Seoul National University, Seoul, Korea, in 1980, and his M.S. and Ph.D. in Electrical Engineering from the Korea Advanced Institute of Science and Technology (KAIST), Daejeon, Korea, in 1986 and 1992, respectively. From 1994 to 1998, he was an Electronics Engineer with Hyundai Electronics Company. In 1993 he was a Research Scientist at the Virginia Polytechnic Institute and State University, Blacksburg. Since 1999 he has been an Associate Professor in the Electronics Engineering Department, Kookmin University, Seoul, Korea. His current research interests are in the areas of modeling and control techniques for power converters as well as EMI analysis and reduction techniques for power electronics circuits.

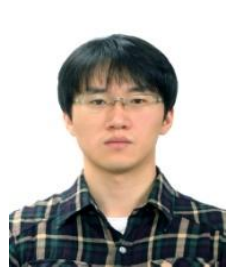

Sang-Ho Cho received his B.S. and M.S. in Electronic Engineering from Kookmin University, Seoul, Korea, in 2006, and 2008, respectively, where he is currently working toward his Ph.D. His current research interests are in the areas of analysis, modeling, design, and control of power converters.

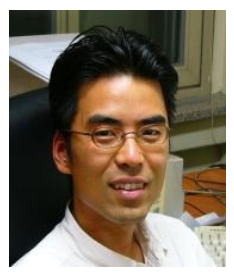

Chung-Wook Roh was born in Busan, Korea, in 1971. He received his B.S., M.S., and Ph.D. in Electrical Engineering from the Korea Advanced Institute of Science and Technology (KAIST), Daejeon, Korea, in 1993, 1995, and 2000, respectively. In 2000, he joined the Digital Media Network Division, Samsung Electronics Company, Suwon, Korea, where he was a Project Leader of the Plasma Display Driver Development Team. Since 2004, he has been an Associate Professor in the Electronics Engineering Department, Kookmin University, Seoul, Korea. His current research interests include driver circuits for plasma display panels, low-power circuits for flat panel displays, modeling, design, and control of power conversion circuits, soft-switching power converters, resonant inverters, and electric drive systems. Dr. Roh is a Member of the Society for Information Display, CA, the Korean Institute of Power Electronics, and the Korean Institute of Electrical Engineers.

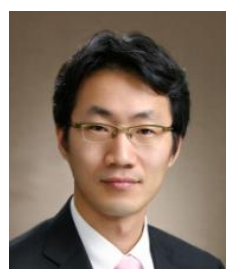

Sang-Kyoo Han was born in Busan, Korea, in 1973 $\mathrm{He}$ received his B.S. in Electrical Engineering from Pusan National University, Busan, Korea, in 1999, and his M.S. and Ph.D. in Electrical Engineering from the Korea Advanced Institute of Science and Technology (KAIST), Daejeon, Korea, in 2001 and 2005, respectively. For six months after that, he worked as a PostDoctoral Fellow at KAIST. Since September 2005, he has been with Kookmin University, Seoul, Korea, as an Assistant Professor in the Department of Electrical Engineering. He has also been working for the Samsung Power Electronics Center (SPEC) and the Samsung Network Power Center (SNPC) at Kookmin University as a Research Fellow. His current research interests are in the areas of power converters, renewable energy systems, and display drivers, including analysis, modeling, design, and control. Dr. Han is a member of KIPE and IEEE. 